\title{
Das „Soziotop“ Jugendstrafanstalt und seine Subkultur
}

Joachim Walter

\section{Die Insassen}

Jugendgefängnisse sind dadurch gekennzeichnet, dass dort eine große Anzahl - meist männlicher - Personen ${ }^{1}$, in der Regel auf engstem Raum und ohne nennenswerte Ausweichmöglichkeit, untergebracht sind und so erzwungener Weise zusammenleben müssen, ob sie sich miteinander verstehen oder nicht. Dass die Insassen des Jugendstrafvollzugs hinsichtlich ihrer sozialen Lage und Herkunft - sie entstammen fast ausnahmslos der Unterschicht bzw. haben einen Migrationshintergrund - im Vergleich zur „Normalbevölkerung “ eine extreme, im Verlauf des Prozesses der Strafverfolgung bewirkte Auslese darstellen, haben zahlreiche kriminalstatistische ${ }^{2}$ und gefängnissoziologische ${ }^{3}$ Untersuchungen ergeben. Denn die dabei erfolgenden justiziellen Ausfilterungs- und Selektionsprozesse sind selbstverständlich immer auch an sozialen Kriterien (wie z.B. Schulabschluss, Arbeitslosigkeit, Wohnsituation) orientiert.

Für die nachfolgende Darstellung kann es allerdings dahingestellt bleiben, ob diese festgestellte soziale Selektion auf höherer Straffälligkeit der Angehörigen unterer sozialer Schichten beruht oder, wofür bedeutend mehr spricht, durch den der strafrechtlichen Sozialkontrolle innewohnenden Ausfilterungsprozess erst hergestellt wird oder ob beide Faktoren zusammen wirken. Jedenfalls verfügen viele der Inhaftierten über eher geringe soziale Kompetenzen. Schon deshalb und wegen der engen räumlichen Unterbringung, aber auch wegen des hohen Anteils von Gefangenen mit Migrationshintergund und ihrer Herkunft aus ganz unterschiedlichen Kulturen, sind Missverständnisse und darauf zurückzuführende Konflikte und Fehlreaktionen geradezu vorprogrammiert.

Hinzu kommt, dass es sich bezogen auf die Insassen um eine reine „Männerwelt“, um einen „homosozialen Raum“ handelt, in dem es für den Einzelnen zum „doing masculinity " keine realistische Alternative gibt. Sich in der Männerwelt zu behaupten erfordert auf der Verhaltensebene den Rückgriff auf Strategien und Männlichkeitssymbole, die Anerkennung im System „hegemonialer Männlichkeit“" versprechen. Die homosoziale Struktur der Inhaftiertengruppe und die Tatsache, dass es sich zum guten Teil um junge Männer handelt, die mit ihren Vor- stellungen von Mann-Sein experimentieren (müssen), lässt Hierarchisierungsprozesse und Rangkämpfe besonders wahrscheinlich und auch leicht sichtbar werden. ${ }^{4}$ Selbst wer durchaus gesonnen ist, sich an die Anstaltsregeln zu halten, wird häufig in die Situation geraten, sich gegen Zumutungen von Mitgefangenen, die versuchen, ein Spielchen mit ihm zu spielen, notfalls brachial zur Wehr setzen zu müssen. So werden Neuzugänge häufig von den „alten Hasen“ Initiationstests unterzogen, die Prüfungscharakter haben und für die Stellung in der Gefangenenhierarchie Bedeutung gewinnen. ${ }^{5}$ In solchen Fällen gibt es auf die Frage, ,wer die Position des Opfers und wer die des Täters einnimmt “, keine eindeutige Antwort. ${ }^{6}$ Ähnliches gilt für die „durchlässige Grenze zwischen Handel und Raub, was den Besitzerwechsel begehrter Objekte betrifft “. ${ }^{7}$ Dabei müssen diese für Gefangene typischen kollektiven Inszenierungen von Härte auch als eine Reaktion auf den Autonomieverlust im Strafvollzug verstanden werden. ${ }^{8}$ Dieser Autonomieverlust, die umfassende Abhängigkeit von der Institution und ihren Repräsentanten, bildet außerdem die Basis für zahlreiche Alltagskonflikte zwischen Gefangenen und Bediensteten, ${ }^{9}$ in denen es um den Versuch geht, sich einen Rest von Selbstbestimmung und auch Selbstachtung zu bewahren. „Unverrückbar erscheint somit die Tatsache, dass sich alle Inhaftierten zu Gewalt in Beziehung setzen müssen - egal ob durch Stärke, Klugheit oder Gewaltbereitschaft “.${ }^{10}$ Sartres Diktum „... die Hölle, das sind die Anderen "11 charakterisiert die Situation im Strafvollzug durchaus treffend.

\section{Das Personal}

Ist auf der einen Seite die Subkultur der Gefangenen zumindest z. T. als Reaktion auf die „totale Institution“12 Gefängnis zu verstehen ${ }^{13}$, so spielt die komplementäre Rolle dazu auf der Seite der Anstalt die der totalen Institution eigentümliche, weit reichende Kontrolle aller Lebensäußerungen und der gesamten organisatorischen Abläufe durch das Personal. Sowohl der Erziehungs- als auch der Sicherungsauftrag der Anstalt erfordern es, dem Grundsatz „Hinsehen, niemals wegschauen" bei den Bediensteten Geltung zu verschaffen. Im Vergleich zum Leben außerhalb ist deshalb die Kontroll- dichte gewaltig erhöht. Neben den vielfältigen Anwesenheits-, Haftraum- und Sicherheitskontrollen mögen als weitere Beispiele die zahlreichen Kontrollen der Gefangenen auf Besitz oder Konsum von Drogen dienen, die durch die Abforderung von Urinproben, Körperkontrollen, Einsatz von Rauschgifthunden bei Haftraumkontrollen und beim Besuch durchgeführt werden. ${ }^{14}$ Man stelle sich vor, Kontrollen von dieser Häufigkeit und Intensität würden, was selbstverständlich nicht vorgeschlagen werden soll, in einem Studentenwohnheim oder in einem First-Class-Hotel durchgeführt. Die „Ausbeute" wäre wohl beträchtlich ...

\section{Mangelwirtschaft}

Auch wenn nach der Vorstellung des Gesetzgebers und nach den europäischen Strafvollzugsgrundsätzen ${ }^{15}$ die Freiheitsstrafe ausschließlich im Entzug der Freiheit bestehen sollte ${ }^{16}$, herrscht auch heute noch in der Jugendstrafanstalt eine ausgesprochene Mangelwirtschaft. Ist bereits die gesamte Lebenshaltung in materieller Hinsicht sehr eingeschränkt, gilt dies noch viel mehr für die Kommunikationsmöglichkeiten mit der Außenwelt, insbesondere mit den Angehörigen oder der Freundin. Die Benutzung des Telefons ist nur ausnahmsweise und unter entsprechender Reglementierung, diejenige des Internet meist überhaupt nicht gestattet. Bargeld ist verboten. Heterosexuelle Kontakte sind außer im Rahmen von Ausgängen oder Urlaub, die zumindest in der Anfangsphase des Vollzuges der Mehrzahl der Gefangenen nicht gewährt werden, so gut wie ausgeschlossen. Homoerotische Kontakte sind im Jugendstrafvollzug wegen der enormen Aversion der ihre sexuelle Identität noch suchenden jungen Männer gegen „die Homosexuellen “ nur in viel geringerem Umfang faktisch möglich, als das oft vermutet wird. Es besteht mithin ein eklatanter Mangel an - gleichwohl ersehnter - Zärtlichkeit.

\section{Folgen}

Es leuchtet ein, dass die beschriebene Situation der Gefangenen im Vollzug, von diesen insgesamt als schwere Deprivation ${ }^{17}$ empfunden, mehr oder weniger zwangsläufig unerwünschte Reaktionen ${ }^{18}$ nach sich ziehen wird: Neben Übersprungs- und Kompensationshandlungen wie z.B. Suicidver- 
suchen, Demolierung der Einrichtung des Haftraums sowie psychischen und psychosomatischen Erkrankungen ${ }^{19}$, die als direkte oder indirekte Auswirkungen der Inhaftierung anzusehen sind, führen die materiellen und kommunikativen Einschränkungen zu Schmuggel und illegalem Handel mit Waren und Dienstleistungen (z.B. Bargeld, Drogen, Pornographie, Mobiltelefone, Briefe). Der Schwarzhandel blüht, Ersatzwährungen ${ }^{20}$ entstehen. Empfundene Frustrationen und das enge Zusammenleben mit „Kameraden", die man sich nicht ausgesucht hat und denen man anderswo aus dem Wege gehen könnte, geben Gründe zu aggressiven Reaktionen, interkulturelle Missverständnisse zu Streitigkeiten und $\mathrm{zu}$ - manchmal ethnisch orientierten - Gruppenbildungen, um nur die häufigsten Folgen aufzuzählen. Vielfach handelt es sich dabei zumindest tatbestandlich um Straftaten wie Diebstahl, Beleidigung, Körperverletzung, Bedrohung, Betrug, Sachbeschädigung, Nötigung und Erpressung, Raub.

Herkömmlich charakterisiert man diese Gegenkultur, die von den Gefangenen dem Deprivationsdruck in der Art eines Abwehrmechanismus entgegengesetzt wird, als die Subkultur des Gefängnisses. ${ }^{21}$ Schon mit dem auch hier benutzten Begriff der Subkultur wird freilich meistens und im selben Atemzug ihre Bekämpfung gefordert. Es wird dann aber, was hier problematisiert werden soll, diese nahe liegende und sehr verständliche Reaktion von Menschen auf Überwachung, Kontrolle und Bestrafung ausschließlich negativ bewertet, ohne dabei auch die Gründe und psychohygienischen Vorteile dieser nahezu unvermeidlichen Reaktionen und Einrichtungen des Selbstschutzes $^{22}$ kritisch zu hinterfragen ${ }^{23}$ und zu prüfen, ob und inwieweit in der gegebenen Situation überhaupt sinnvolle Handlungsalternativen bestehen. Denn subkulturelles Verhalten kann in totalen Institutionen als „sekundärer Anpassungsmechanismus“ u.U. die einzige Möglichkeit für Insassen sein, einen Rest von Selbstbestimmung zu bewahren. $^{24}$

Will man als Gefangener „einigermaßen über die Runden kommen “, wird man sich wenigstens an die wichtigsten Regeln der Insassensubkultur halten müssen. Dazu gehört in erster Linie, andere Gefangene in Befragungen nicht gegenüber dem Vollzugspersonal oder der Polizei zu verraten oder gar anzuzeigen, aber auch, den Angehörigen der eigenen Bezugsgruppe oder Clique zu helfen (z.B. als Mannheimer anderen Mann- heimern, als Albaner seinen Landsleuten) und im Streitfall aktiv beizustehen. Wie die Menschen außerhalb auch sind die Gefangenen in ihre Umgebungskultur eingebunden. Nur enthält die den Gefangenen aufgenötigte Lebensweise ${ }^{25}$ ganz erhebliche Abweichungen vom freiheitlichen Leben, weshalb sie sich situativ und im eigenen verständlichen Interesse oft kaum anders verhalten können, ${ }^{26}$ als es der „second code“ 27 , die subkulturelle Norm, gebietet. ${ }^{28}$ Auch aus der Sicht von außen wird man ihnen oft keinen besseren Rat geben können. Wurden beispielsweise einem Gefangenen von zwei anderen gemeinschaftlich Ohrfeigen angedroht, so verstößt er als Zeuge vor Gericht gegen seine Aussagepflicht, wenn er dies wahrheitswidrig verneint, und riskiert eine Bestrafung wegen Falschaussage - solche Verurteilungen sind vorgekommen! Sagt er aber wahrheitsgemäß aus, hat er unter allen seinen Mitgefangenen Gesicht und Rang verloren und wird ihm zukünftig als „Anscheißer“ das Leben schwer gemacht werden. „Orientieren die Jugendlichen ihre Handlungsstrategien am Codex der Eigengruppe, geraten sie über kurz oder lang in Konflikt mit dem Codex des Vollzuges; lassen sie sich auf den Regelkanon des Vollzuges ein, laufen sie Gefahr, von anderen Inhaftierten ausgegrenzt zu werden. “29 Zumindest aus ihrer Sicht ein unlösbares Dilemma, das man in Anlehnung an Treibers Begriffsschöpfung ${ }^{30}$ als atypische Normenfalle bezeichnen kann. Es gibt also tatsächlich, wie es Verrel formuliert hat, „Delikte, die man als unvermeidbare Begleiterscheinungen von Binnengesellschaften wie dem Strafvollzug bezeichnen kann“31. Dies wirft die Frage auf, wie man mit dieser besonderen Situation umgehen soll.

\section{Erhöhte Sanktionsgefahr durch hohe Kontrolldichte}

Neben die durch die hoch selektierte Population der Strafanstalt und die Deprivation der Gefangenen ohnehin erhöhte Belastung mit Straftaten tritt noch eine durch die hohe Kontrolldichte verursachte bessere Ausleuchtung des bekanntlich enorm großen Dunkelfeldes $^{32}$, jedenfalls dann, wenn das Vollzugspersonal seine Überwachungsaufgaben im oben beschriebenen Sinne erfüllt. Davon kann aber nicht nur deshalb ausgegangen werden, weil Vollzugsbeamte nach Nr. 9 DSVollz verpflichtet sind, der Anstaltsleitung alle wichtigen Beobachtungen mitzuteilen, sondern auch ausweislich der in nahezu jeder Personalakte eines Gefange- nen vorhandenen - meist disziplinarischen - Meldungen. Das Entdeckungsrisiko für normabweichendes Verhalten jeder Art ist $\operatorname{hoch}^{33}$, freilich abhängig davon, wie viel und wie gut ausgebildetes Personal zum Einsatz kommt, außerdem auch davon, wie gut es gelingt, Informationen aus der und über die Subkultur zu erlangen.

Aufgrund der drei bisher beschriebenen, so nur unter Vollzugsbedingungen zusammen kommenden Faktoren:

- Vorselektion der Population nach Kriterien der sozialen und kulturellen Herkunft sowie abweichenden Verhaltens,

- mehr oder weniger starke Einbindung in die Gefangenensubkultur,

- enorme Kontrolldichte und damit bessere Aufhellung des Dunkelfeldes

ergeben sich nun weitreichende, ja fast unerschöpfliche Möglichkeiten für negative Sanktionen: Sowohl für formelle Disziplinierungen durch die Anstaltsleitung auf der einen Seite wie auch für strafrechtliche Verfolgung durch Polizei und Staatsanwaltschaft auf der anderen Seite. ${ }^{34}$ Immerhin sind an keinem anderen Ort so viele strafrechtlich vorbelastete Menschen konzentriert und besteht nirgendwo sonst eine derart hohe Kontrolldichte wie im Strafvollzug. Um es in einem Bild auszudrücken: Der Schwarm der von vornherein Verdächtigen und Vorbelasteten ist groß; und angesichts der engen Begrenztheit des Gewässers kann der Einzelne dem Kescher kaum entgehen.

\section{Zweck der Überwachung?}

Bevor man auf repressive Sanktionierung subkulturellen Verhaltens zielenden Überlegungen weiter verfolgt, sollte man sich die Frage vorlegen, welchen Zwecken die im Jugendstrafvollzug durchgeführten Aufsichtsmaßnahmen eigentlich dienen? Da ist selbstverständlich die Aufrechterhaltung der Ordnung in der Anstalt. Die Aufsicht und die Kontrollen haben zunächst präventive Zwecke: Das Ziel der Fluchtverhinderung, ebenso, Übergriffe unter Gefangenen sowie andere Straftaten zu verhindern. Sie streben zunächst nichts Anderes an, als einen funktionsfähigen Strafvollzug zu gewährleisten.

Des Weiteren ist im modernen Jugendstrafvollzug die Aufsicht natürlich auch dem Resozialisierungsziel verpflichtet. Dieses, unstreitig ein Leben ohne Straftaten in sozialer Verantwortung, ist wesentlich zukunftsorientiert. Auch hieraus ergibt sich, dass der Zweck der Aufsicht im Resozialisierungs- 
und Erziehungsvollzug nicht in erster Linie in der Ermittlung und Ahndung von Straftaten, sondern in ihrer Verhinderung liegt, ggf. auch ihrer pädagogischen Aufarbeitung, notfalls mit den Mitteln des vollzuglichen Disziplinarrechts.

Auch wenn im Jugendstrafvollzug Straftaten häufig vorkommen und vom Personal registriert werden, geht es dort primär nicht darum, Gefangene strafrechtlich zur Verantwortung zu ziehen, sondern sie zu befähigen, ein Leben ohne Straftaten in sozialer Verantwortung zu führen. Dazu kann es gehören, dass verübte und entdeckte Delikte strafrechtlich sanktioniert werden. Da die Justizvollzugsanstalt aber unzweifelhaft keine Strafverfolgungsbehörde ist, obliegen ihr insoweit auch keine anderen Aufgaben, als gegebenenfalls die zuständigen Strafverfolgungsorgane einzuschalten. Ansonsten handelt es sich hier um Aufgaben der Polizei, der Staatsanwaltschaft und anderer Strafverfolgungsbehörden. Im Übrigen haben Vollzugsbedienstete in dieser Hinsicht auch nicht die erforderliche Ausbildung. Ungeachtet ihrer Dienstpflicht, die Anstaltsleitung über wichtige Wahrnehmungen zu informieren, sollten sie sich eigener Ermittlungshandlungen zum Zwecke der Strafverfolgung enthalten.

\section{Was tun?}

In der und durch die Subkultur findet eine zunehmende Identifizierung der Gefangenen mit illegitimen Lernstrukturen und Rollenangeboten statt. Versucht man hiergegen mit repressiven Maßnahmen - z.B. Disziplinarmaßnahmen oder Strafanzeigen - vorzugehen, besteht die Gefahr, dass die Subkultur sogar noch verstärkt wird, weil im kollektiven Schutz der Gefangenengruppe Abwehrkräfte gegen Sanktionen besser entwickelt werden können. Hoher Druck zwingt die Insassen gleichsam in die Subkultur sowie dazu, illegitime Methoden zu erlernen. Wird beispielsweise ein Gefangener aus disziplinarischen Gründen damit bestraft, dass er zeitweise am Anstaltseinkauf nicht teilnehmen kann, besteht die Gefahr, dass er nach Möglichkeiten suchen wird, sich seinen notwendigen Bedarf an Tabak u.a. durch Erpressung Schwächerer zu beschaffen.

Durch im Jugendstrafvollzug leider nicht seltene Überreglementierung kann die Situation noch weiter dadurch verschärft sein, dass der Insasse einer solchen Vielzahl von Verhaltensanforderungen und Normen aus- gesetzt ist, dass er, selbst wenn er das wollte, unmöglich alle beachten kann: Er sitzt in der „Normenfalle“ ${ }^{35}$. Er ist sozusagen zum Normbruch gezwungen und dadurch auch ständig in Gefahr, sanktioniert zu werden. Das wiederum verstärkt den subkulturellen Zusammenhalt der Gruppe, am Ende sogar die Gefahr von Gewalttätigkeit.

Theoretische Überlegungen wie empirische Befunde legen nahe, dass unterschiedlich repressive Vollzugsformen die Gefängnissubkultur verstärken oder verringern können. Wie zahlreiche frühere Untersuchungen hat Grosch in einer Studie für den süddeutschen Jugendstrafvollzug gefunden, dass bei Gefangenen ohne Vollzugslockerungen die subkulturelle Orientierung sowie die Belastung mit Disziplinarmaßnahmen zur Mitte der Haftzeit hin ihr Maximum erreicht, wogegen umgekehrt bei Gefangenen, die zu diesem Zeitpunkt Vollzugslockerungen erhielten, deviantes Anstaltsverhalten abnahm und einen Tiefpunkt erreichte ${ }^{36}$. Für den offenen Jugendstrafvollzug (in Flensburg) war Sonnen ${ }^{37}$ zu dem Ergebnis gekommen, dass dort „eine Auslieferung an die Statushierarchie der Gefangenen, ihre Prozesse der Machtbildung, an Abhängigkeit, das Handelssystem mit Ware und Dienstleistung und eine Anpassung nicht an Gesellschaftsnormen, sondern an die Insassensubkultur nicht stattfindet“. Ein Vergleich zwischen Häusern des geschlossenen Vollzuges und solchen des intern gelockerten Vollzuges in der Justizvollzugsanstalt Adelsheim hat ähnliche Ergebnisse erbracht ${ }^{38}$. Aus alledem ergibt sich, dass im Hinblick auf das Ausmaß und die Bedeutung der Subkultur zwischen offenen und geschlossenen Vollzugsformen erhebliche Unterschiede bestehen.

Als Kurzformel kann daher gelten: Je offener der Vollzug, desto weniger Subkultur. Werden also Prisonisierungseffekte (im Sinne subkultureller Prägung des Einzelnen) durch Außenkontakte und insbesondere Vollzugslockerungen vermindert, kommt man um die Schlussfolgerung nicht herum, dass der resozialisierungsfeindlichen Insassensubkultur am besten durch eine Änderung der formellen Anstaltsstruktur in Richtung auf Öffnung des Vollzuges beizukommen sein wird, wenn auch individuelle Therapie, Sozialarbeit, Sport, schulische und berufliche Ausbildung, um nur einige zu nennen, unterstützend wirken können ${ }^{39}$. Wer etwas zum Abbau der gefängnistypischen Subkultur beitragen will, muss bedenken, dass ein „hartes Regime“ im Gefängnis oppositionelle Insasseneinstellungen eher noch begünstigt, und damit ein Nährsubstrat für die Bildung der Insassensubkultur liefert. Er sollte deshalb die altbekannten repressiven Sanktionen zurückfahren und ihnen Maßnahmen in Richtung auf eine strukturelle Öffnung des Gefängnisses zur Seite stellen.

Dr. Joachim Walter war bis Ende 2009 Leiter der Jugendstrafanstalt Adelsheim, arbeitet nun als Rechtsanwalt und ist Mitherausgeber dieser Zeitschrift.

\section{Fußnoten:}

1 Bei rund $94 \%$ der Jugendstrafgefangenen handelt es sich um Männer. Die besondere Situation in den wenigen Anstalten für junge Frauen in der Bundesrepublik Deutschland und ihre durchaus differente Subkultur bleibt hier außer Betracht.

2 Näher hierzu z.B. Heinz, W. (2006): Kriminelle Jugendliche - gefährlich oder gefährdet? Konstanz, S. 29 ff. Vgl. auch das bekannte „Trichtermodell“, wonach (im Jahre 2008) nur ca. $1 \%(!)$ der von der Polizei wegen gefährlicher oder schwerer Körperverletzung ermittelten Jugendlichen in den Strafvollzug gelangten, wohingegen bei allen anderen das Verfahren eingestellt wurde oder sie zu einer nicht freiheitsentziehenden Sanktion verurteilt wurden (Konstanzer Inventar Sanktionsforschung; www.ki.uni-konstanz.de/kis).

3 Für Deutschland etwa Enzmann, D. und Greve, W. (2001): Strafhaft für Jugendliche: Soziale und individuelle Bedingungen von Delinquenz und Sanktionierung, in : Bereswill, M. /Greve, W. (Hrsg.:) Forschungsthema Strafvollzug, S. 109

4 Bereswill, M. (1999): Gefängnis und Jugendbiographie. Qualitative Zugänge zu Jugend, Männlichkeitsentwürfen und Delinquenz. KFN Forschungsberichte Nr. 78, S. $10 \mathrm{f}$

5 Vgl. Bereswill, M. (2007): Die einschneidende Erfahrung einer Inhaftierung im Jugendstrafvollzug, S. 173 f. In: Goerdeler, J. / Walkenhorst, Ph. (Hrsg.): Jugendstrafvollzug in Deutschland. Neue Gesetze, neue Strukturen, neue Praxis? Mönchengladbach 2007.

6 Bereswill, M. (2001): „Die Schmerzen des Freiheitsentzuges“ - Gefängniserfahrungen und Überlebungsstrategien männlicher Jugendlicher und Heranwachsender in Strafhaft. In: Bereswill, M. / Greve, W. (Hrsg.): Forschungsthema Strafvollzug, 1. Auflage Baden-Baden, S. 272 f.

7 Bereswill aaO S. 277 verweist in diesem Zusammenhang auf die schon von Goffman (1973, S. 30, vgl. unten Fußnote 16) beschriebene komplementäre Strategie der Institution Strafvollzug, die Gefangenen zu ent-individualisieren, indem sie ihre privaten Besitztümer abgeben müssen.

8 Bereswill, M. aaO S. 281

9 Vgl. Bereswill (Fußnote 9) S. 172

10 Neuber, A. (2007): Anti-Gewalttrainings und biographische Zugänge zu Gewalt, S. 262. In: Goerdeler, J. / Walkenhorst, Ph. (Hrsg.): Jugendstrafvollzug in Deutschland. Neue Gesetze, neue Strukturen, neue Praxis? Mönchengladbach 2007.

11 Sartre, J. P.: Bei geschlossenen Türen. In: Gesammelte Dramen. Reinbek bei Hamburg 1969, S. 97

12 Goffman, E. (1973): Asyle Frankfurt a.M.; Pecher, W. (2004): Totale Institution, S. $310 \mathrm{ff}$, in: Pecher, W. (Hrsg.): Justizvollzugspsychologie in Schlüsselbegriffen, Stuttgart.

13 Sog. „Deprivationstheorie“. Da aber nicht selten auf Verhaltensmuster aus anderen Subkul- 
turen außerhalb des Gefängnisses, beispielsweise der Drogensubkultur, zurückgegriffen wird (sog. „Importtheorie“ oder „Theorie der kulturellen Übertragung “), sprechen die Ergebnisse der Strafvollzugsforschung in Deutschland wie auch die praktische Erfahrung dafür, beide Theorien zu vereinigen (Dünkel, F.: Empirische Forschung im Strafvollzug. Bestandsaufnahme und Perspektiven. Bonn 1996, S. 4 ff mwN).

14 Möglicherweise in Folge dieser häufigen Kontrollen pflegt die Zahl der des Drogenkonsums überführten Gefangenen und erst recht der beschlagnahmten Drogen eher gering zu sein. So wurde beispielsweise in der Jugendstrafanstalt Adelsheim im Jahre 2006 bei nicht weniger als 565 durchgeführten Urinkontrollen in 38 Fällen BTM-Konsum nachgewiesen, davon in 34 Fällen Cannabisprodukte.

15 Europäische Strafvollzugsgrundsätze vom 12. 2. 1987, Nr. 64; ebenso die schon 1955 in Genf verabschiedeten UN Standard Minimum Rules for the Treatment of Offenders, No. 57.

16 Walter, M. (Fußnote 1) Rz. 22 f, 51; Baechtold, A. (2005): Strafvollzug. Bern, S. 37. Kritisch zu diesem Konzept Chantraine, G.: Les temps des prisons. Inertie, réformes et reproduction d'un dispositif institutionnel. In: Artières, Ph. und Lascoumes, P. (Ed.): Gouverner, enfermer. La prison, un modèle indépassable, Paris 2004, S. 69: „L'utopie d'une pénalite incorporelle, qui voudrait que la prison soit une privation de liberté et rien d'autre, se heurte, à travers les époques, à la poussière des faits concrets".

$17 \mathrm{Zu}$ Haftdeprivationen Laubenthal, K. (2007): Strafvollzug, 4. Aufl. Berlin, Heidelberg, New York, Rz. 209 ff.

18 Das hat der Gesetzgeber seit langem erkannt und deshalb im sog. Gegensteuerungsgrundsatz ( 3 Abs. 2 StVollzG) den Vollzugsbehörden aufgegeben: „Schädlichen Folgen des Freiheitsentzuges ist entgegenzuwirken“.

19 Eine aktuelle Übersicht hierzu m.w.N. bei Drenkhahn, K. / Dudeck, M. (2007): Lebensbedingungen im europäischen Langstrafenvollzug. Neue Kriminalpolitik 4/2007, S. 134-138.

20 wie „Koffer“ (1 Päckchen Tabak) oder „Bombe" (1 Glas Pulverkaffee).

21 Harbordt, S. (1972): Die Subkultur des Gefängnisses, Stuttgart.

22 Walter, M. (Fußnote 1), Rz.266: „Da jedoch schon die Existenz eines Gefängnisses für die dorthin Eingelieferten eine Fülle von Identitätsangriffen und -gefährdungen mitbedingt, dürfte die Bildung von abwehrenden informellen Systemen der Gefangenen kaum vermeidbar sein".

23 Müller-Marsell, S. (2004): Subkultur im Strafvollzug, in: Pecher, W. (Hrsg.): Justizvollzugspsychologie in Schlüsselbegriffen, S. 286 ( 287).

24 Goffman, E. (Fußnote 15) S. 59 f: „Manchmal wird die sekundäre Anpassung nachgerade zu einem Bollwerk des Selbst, zu einer Art Beschwörungsformel, in der die Seele wohnt".

25 Eisenberg ZJJ 2008, S. 381: „Staatlich organisierte Unentrinnbarkeit“.

26 Ähnlich Walter, M. (1999): Strafvollzug, 2. Aufl., Rz. 261, 264, 266.

27 MacNaughton-Smith, P. (1975): Der zweite Code. Auf dem Weg zu einer (oder hinweg von einer) empirisch begründeten Theorie über Verbrechen und Kriminalität. In: Lüderssen, K. / Sack, F. (Hrsg.): Seminar abweichendes Verhalten II, Frankfurt a.M., S. 197.

28 Eisenberg aaO: „Die Funktion subkultureller Normen schlechthin und im Speziellen des zumindest partiell endemischen Wesens der Insassen-Subkultur (...) liegt aber darin begründet, dass sie gegebenenfalls von der Einzelperson losgelöst wirken, und zwar erhöht bei gruppendynamischem Geschehen."

29 Bereswill aaO S. 275.

30 Sog. „Normenfalle“. Treiber, H.: Wie man Soldaten macht. Sozialisation in kasernierter Vergesellschaftung. Düsseldorf 1973, S. 43.

31 Verrel, T. (2003): Der Anstaltsleiter als Garant für die Verfolgung von Straftaten während des Strafvollzugs? Goltdammers Archiv S. 595 $-609$

32 Nach Kerner, H.-J., Jugendkriminalität zwischen Massenerscheinung und krimineller Karriere - Eine Problemskizze an Hand neuerer Statistischer Ergebnisse, in: Nickolai, W. / Reindl, R. (Hrsg.): Sozialarbeit und Kriminalpolitik. Freiburg 1993, S. 29 geben im Schnitt „über 90\% der mit Befragungen erfassbaren Jungen und jungen Männer an (bzw. zu), mindestens einmal im ihren seitherigen Leben, regelmäßig jedoch wiederholt, Handlungen begangen zu haben, die juristisch unter eine Strafnorm ... subsumiert werden könnten“.

33 Kühnel, W. (2007): Gruppen, Konflikte und Gewalt im Jugendstrafvollzug. APuZ 37/2007

34 Näher Walter, J.: Formelle Disziplinierung im Jugendstrafvollzug. Frankfurt, Berlin usw. 1997

35 Treiber (FN 30), S. $43 \mathrm{ff}$

36 Grosch, O. 1995: Lockerungen im Jugendstrafvollzug. (Kriminologische Forschungsberichte aus dem Max-Planck-Institut für Ausländisches und internationales Strafrecht; Bd. 48) Freiburg, S. $368 \mathrm{ff}$

37 Sonnen, B.-R. 1994, Der offene Jugendstrafvollzug in Flensburg (Begleitforschungsbericht). DBH Materialien Nr. 18, S. 44

38 Näher J. Walter (FN 34), S. $111 \mathrm{ff}$

39 Harbordt, (FN 21), S. 100

\section{Wie geht es 2012 weiter mit der Reform der Sicherungsverwahrung?}

Am 17. November 2011 hat das BMJ seinen Referentenentwurf an betroffene Fachkreise und Verbände mit der Bitte um Stellungnahme übermittelt, Heinz Cornel wird in Heft 1-2012 mit der Kommentierung beginnen. Nach $\$ 66 \mathrm{~b}$ (Nachträgliche Anordnung der Unterbringung in der Sicherungsverwahrung) soll ein neuer $\$ 66 \mathrm{c}$ mit dem Titel „Ausgestaltung der Unterbringung in der Sicherungsverwahrung und des vorherigen Strafvollzugs“ eingefügt werden. Danach ist nach den Vorgaben des BVerfG vom 4. Mai 2011 (2 BvR 2365/09) künftig folgendes zu beachten:

- dem Untergebrachten Betreuung anzubieten auf der Grundlage einer umfassenden Behandlungsuntersuchung und eines regelmäßig fortzuschreibenden Vollzugsplans,

- er ist individuell und intensiv zu betreuen (Individualisierungs- und Inensivierungsgebot), die Mitwirkungsbereitschaft des Sicherungsverwahrten ist zu wecken und zu fördern (Motivierungsgebot), ferner sind anzubieten psychiatrische, psycho- oder sozialtherapeutische Behandlungen, welche

- zum Ziel haben, die Gefährlichkeit der Verwahrten für die Allgemeinheit so zu mindern, dass die Vollstreckung der Maßregel möglichst bald zur Bewährung ausgesetzt oder sie für erledigt erklärt werden kann,

- eine Unterbringung gewährleisten, die den Untergebrachten so wenig wie möglich belastet und, soweit Sicherheitsbelange nicht entgegenstehen, den allgemeinen Lebensverhältnissen angepasst ist,

- in der Regel vom Strafvollzug getrennt sind und sich in besonderen Gebäuden oder Abteilungen befinden (Trennungsgebot),

- vollzugsöffnende Maßnahmen gewähren und Entlassungsvorbereitungen treffen, soweit nicht zwingende Gründe entgegenstehen, insbesondere die konkrete Gefahr besteht, dass sich der Untergebrachte dem Vollzug entziehen oder die Maßnahmen zur Begehung schwerer Straftaten missbrauchen wird und

- Nachsorge in Kooperation mit staatlichen und freien Trägern ermöglichen.

Der Entwurf legt damit kriminalpolitisch die Grundlagen für eine neue Ausrichtung der Sicherungsverwahrung, wie sie vom BVerfG insbesondere als Mindestaspekte eines Gesamtkonzeptes gefordert wurden. Das ist zweifellos kein Abschied von und auch keine wirkliche Einschränkung der Anwendung der Sicherungsverwahrung, die das BVerfG aber auch nicht gefordert hatte. Hinsichtlich der Ausgestaltung der Unterbringung zeichnet sich der Referentenentwurf nach der ersten Einschätzung von Heinz Cornel weitgehend durch Konsequenz und Stimmigkeit aus. 